\title{
Pore Network Modeling to Study the Impacts of Geometric Parameters on Water Transport inside Gas Diffusion Layers
}

\author{
H. Gholipour ${ }^{1}$, M. J. Kermani ${ }^{1 \dagger}$ and R. Zamanian ${ }^{2}$ \\ ${ }^{1}$ Department of Mechanical Engineering, Amirkabir University of Technology, Tehran, 15875-4413, Iran \\ ${ }^{2}$ International Campus, Amirkabir University of Technology, Tehran, 15875-4413, Iran \\ †Corresponding Author Email: mkermani@aut.ac.ir
}

(Received November 12, 2020; accepted June 6, 2021)

\begin{abstract}
A pore network model (PNM) is proposed for the simulation of water transport inside the cathode side gas diffusion layer (GDL) of polymer electrolyte fuel cells (PEFCs) during the transient start-up period as well as the steady state. Numerous two-dimensional random networks representing GDL are generated followed by statistical averaging of the results (Monte Carlo methods) to circumvent the uncertainties imposed by random pore size distributions. The resulting liquid water saturation profiles within GDLs exhibit concave patterns which is typically encountered in capillary fingering flow regimes in porous media. The effect of GDL thickness and current collector rib width as two geometric parameters on water transport dynamics are separately investigated. It turns out that thin and thick GDLs compared to the base case can have contradicting outcomes on the account of total water saturation in the network. On the other hand, wide current collector ribs give rise to liquid water saturation and build-up within GDL which can lead to flooding. At the end, three-dimensional networks are generated demonstrating higher pore connectivity which results in higher percolation times and different invasion patterns.
\end{abstract}

Keywords: Fuel cells; Gas diffusion layer; Two-phase flow; Pore network modeling; Capillary fingering.

NOMENCLATURE

$\begin{array}{ll}A_{a} & \text { flow cross-section of air } \\ A_{\mathrm{duct}} & \text { pore (duct) cross section } \\ A_{\mathrm{W}} & \text { flow cross-section of water } \\ H & \text { GDL thickness } \\ i & \text { current density } \\ L & \text { pore length } \\ P_{\mathrm{a}} & \text { air pressure } \\ P_{\mathrm{w}} & \text { liquid water pressure } \\ P_{\mathrm{c}} & \text { capillary pressure } \\ Q_{i j} & \text { volume flow rate in the pore between nodes } i \text { and } j \\ R_{\mathrm{P}} & \text { pore size (radius of the inscribed circle) } \\ R H & \text { relative humidity } \\ R i b & \text { width of the two half-ribs } \\ S_{\text {tot }} & \text { total saturation } \\ S_{Y} & \text { local saturation along the } Y \text { direction } \\ t & \text { time } \\ t_{0} & \text { initial time }\end{array}$

$\begin{array}{ll}V(x, t) & \text { velocity } \\ V_{i j} & \text { velocity in pore } i j \\ V_{\mathrm{P}} & \text { void volume of the network } \\ V_{\mathrm{tot}} & \text { total volume of the network } \\ X_{0} & \text { initial position of the meniscus } \\ X_{i j} & \text { meniscus position inside the pore } \\ \beta & \text { Weibull PDF shape parameter } \\ \gamma & \text { surface tension } \\ \varepsilon_{\mathrm{n}} & \text { nominal porosity } \\ \eta & \text { Weibull PDF scale parameter } \\ \theta_{\mathrm{a}} & \text { contact angle of air } \\ \theta_{\mathrm{w}} & \text { contact angle of water } \\ \lambda & \text { random number } \\ \mu_{\mathrm{a}} & \text { viscosity of air } \\ \mu_{\mathrm{w}} & \text { viscosity of water } \\ \mu_{\mathrm{eff}, i j} & \text { effective viscosity } \\ \xi & \text { Weibull PDF location parameter }\end{array}$




\section{INTRODUCTION}

Polymer electrolyte fuel cells (PEFCs) are generally believed to emerge as the next generation of engines to be used in automotive industry and as alternatives to internal combustion engines. There is a severe competition between fuel cells and batteries, each having their own advantages and disadvantages. PEFCs as a leading type of fuel cells have some disadvantages which put obstacles on the way of their implementation; size, cost, water management and power density are some of the main ones which must be circumvented before their commercialization (O'Hayre et al. 2016).

A PEFC is composed of an anode side and a cathode side; every side contains a porous gas diffusion layer (GDL) and a catalyst layer (electrochemical reaction site). A Nafion membrane exists at the central part which is sandwiched by the two sides. Water and heat are the only reaction products which means PEFCs generate electricity with almost zero greenhouse emission. The porous medium in GDL is traversed by gaseous reactants, product water and electrons between catalyst layer and bipolar plates (comprising gas channels (GCs) and the current collector ribs). Water is generated during the cathode side half reaction in the cathode catalyst layer (CCL); in an attempt to have GDL void volume free for oxygen diffusion, the product water is required to be removed as vapor, but it necessitates dry oxygen delivery that may result in membrane dehydration and high ohmic overvoltages. By contrast, liquid water presence within GDL could hinder oxygen access to reaction sites which is more pronounced at elevated current densities; this can cause GDL flooding and loss of efficiency in PEFCs. As a result, maintaining a trade-off between flooding of GDL and membrane dehydration is favorable which is recognized as water management (Li et al. 2008). One common practice which is employed for better removal of water is to coat GDL surface using PTFE which makes the pores hydrophobic.

To elucidate the issue of water transport inside GDL, countless number of numerical investigations have previously been executed. A multitude of the investigations employ the traditional continuum twophase flow models which define average quantities over representative elementary volumes (REVs) (Weber et al. 2014). Nevertheless, the appropriateness of this approach for GDLs is seriously under doubts by some researchers (Rebai and Prat 2009; Tavangarrad et al. 2018); the rationale is that a REV which satisfies the requirements of volume-averaging is nearly as large as the GDL thickness, and in addition, the methodology is not capable of capturing the morphological heterogeneities of GDL microstructure. To mention some more precise works, García-Salaberri et al. $(2015,2018)$ carried out some quantitative analyses of REV definition in carbon fiber papers (CFPs) and GDLs either in terms of GDL morphologies or effective transport properties in CFPs. Both the methods resulted in the verdict that REVs cannot be defined for thin porous materials such as CFPs and GDLs.

As mentioned earlier, GDL is a hydrophobic medium in which water is the non-wetting phase and air is the wetting phase with water transport being a flow regime dominated by capillary effects; GDL also exhibits anisotropic morphology and wettability. To take into account all these phenomena, a pore network model (PNM) is an appropriate choice. The principal notion of a PNM lies in converting a tortuous porous structure to a plain pore and throat network (Aker et al. 1998; Singh and Mohanty 2003); as a result, all possible types of transport phenomena including water transport may be addressed in the network. PNMs are being used for PEFCs for about a decade, in the way that they have been mostly implemented to model two-phase flow inside GDLs (Lee et al. 2009; Rebai and Prat 2009; Lee et al. 2010; Wu et al. 2010; Medici and Allen 2010; Wu et al. 2012; Lee et al. 2014; Fazeli et al. 2015; Qin 2015; Aghighi et al. 2016; Qin et al. 2019; Carrere and Prat 2019; Zhan et al. 2019). Nearly all of these pore network models are predicated on liquid water injection from GDL/CCL interface into GDL. The models successfully determined saturation profiles of liquid water, capillary pressure and relative permeability in terms of saturation, and also explored the influence of non-uniform wettability on two-phase flow.

The majority of the mentioned PNMs utilized the quasi-static invasion-percolation model which is easy to implement. However, it is a statistical model that cannot accommodate any physical time and consequently not able to capture transient behavior. As an alternative, a dynamic PNM not having these shortcomings can subsume viscous phenomena too, however the computational cost is substantial. Some of the researchers employed dynamic PNMs to be applied to water transport in GDLs (Lee et al. 2009; Medici and Allen 2010; Qin 2015; Qin et al. 2019; Gholipour et al. 2021a; b). For instance, Medici and Allen (2010) utilized a two-dimensional (2D) dynamic PNM for GDL to investigate the effect of wetting properties and GDL morphology on water transport. They explored the effect of GDL wetting properties by changing the contact angle; they also examined the effects of different pore size distributions and the presence of microporous layer on liquid water movement. Lee et al. $(2009,2010,2014)$ established quasi-static as well as dynamic PNMs of two-phase flow in GDL; they were able to investigate in their works the effect of different inlet boundary conditions and also some geometric parameters such as current collector rib width on liquid water transport. Qin (2015) and Qin et al. (2019) came up with dynamic pore network models with the inclusion of wetting film pressure computations; they included phase change effects in their model and also highlighted the liquid water separation in under the ribs and under GC regions in GDL. However, in the pore network modeling literature, there seems to be a lack of explicit 
illustration of liquid water configurations in GDL as a result of different geometric parameters.

In this paper, a dynamic pore network model for the gas diffusion layer at the cathode side of PEFCs is proposed. At first, 2D networks are generated to illustrate liquid water saturation distributions inside GDL. To gain better understanding, local saturation profile and also capillary pressure profile evolutions are presented for the flow from the initiation of water injection till reaching steady state. The effect of GDL geometric parameters namely GDL thickness and current collector rib width on water saturation distribution patterns and capillary pressure are investigated afterwards. At the end, two 3D networks are generated which are expected to exhibit different invasion patterns and also illustrate features which are not detectable through 2D networks.

\section{PORE NETWORK CONSTRUCTION}

As the first step of the simulation, a pore network configuration should be generated being able to suitably demonstrate pore structure of commercial GDLs. With regard to the modeling aims, any extent of pore structure details can be deemed for creating the network. Resorting to a topologically equivalent pore network can be laborious being a separate research area on its own. By contrast, a structured network possessing the same general properties such as permeability and porosity would result in the same trends as the complicated topologically equivalent network. The objective in the present research is the generation of a pore network to study the effect of GDL/GC-rib geometry on water transport, so a simple structured network can serve the purpose as most of the mentioned previous works.

Researchers developing structured PNMs, generally employ two distinct methodologies to symbolize the porous medium empty volume. One approach transforms the lattice to pores and throats in which the throats are assumed without volume and all the lattice empty volume is assigned to pores. Another approach is based on tubes and nodes in which all the lattice void volume is allocated to tubes. In this paper, the second approach is taken into account, as a result the void space of GDL is allocated to the tubes connecting volumeless nodes. The tubes are assumed to be ducts of quadrilateral cross-section all having an identical length $L=25 \mu \mathrm{m}$. As of now, the word "pore" signifies a duct between two adjacent nodes. The network is a $2 \mathrm{D}$ one in which 4 ducts intersect at a node; consequently, the coordination number of the network (the average number of passages connected to a node) would be 4 . For a 3D network, the coordination number would be at least 6 . The pore size is specified using its inscribed circle radius $\left(R_{\mathrm{P}}\right)$ in the quadrilateral cross-section by which random volumes can be assigned to pores. To allocate random volumes to the pores, the pore radii $\left(R_{\mathrm{P}}\right)$ are scattered in accordance with a 3-parameter Weibull probability density function (PDF), specifically:

$R_{\mathrm{P}}=\xi+\eta\left[-\ln \left(\frac{1}{1-\lambda}\right)\right]^{\frac{1}{\beta}}$

in which $\lambda$ is a random number which is uniformly distributed in the interval $(0,1), \eta, \beta$ and $\xi$ are scale parameter, shape parameter and location parameter in the Weibull PDF, respectively. The Weibull parameters are determined as $\xi=3, \eta=7$ and $\beta=3$. Based on the Weibull parameters, the sizes of the pores are dispersed in the interval $(3 \mu \mathrm{m}, 15 \mu \mathrm{m})$, and the average pore size of the whole network is $8.2 \mu \mathrm{m}$. The distribution of pore sizes is displayed in Fig. 1, as it was expected, the distribution is not uniform, instead average-sized pores have higher frequencies and are more likely to occur anywhere in the network.

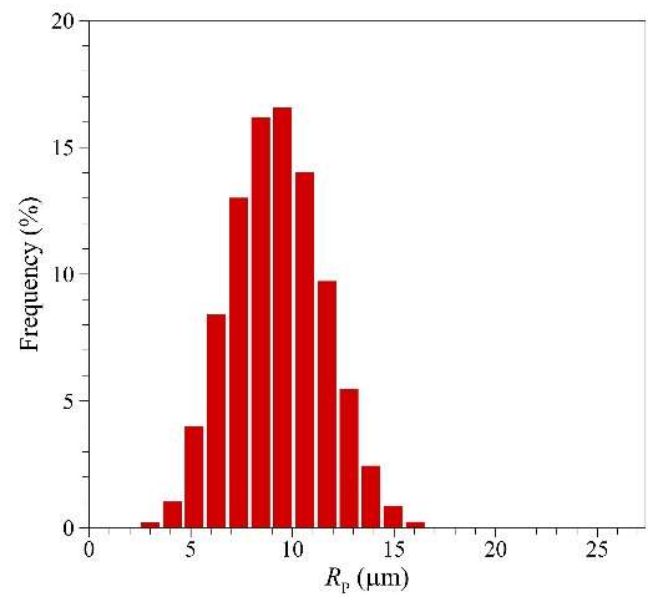

Fig. 1. Frequency distribution of pore sizes according to the Weibull PDF.

To lower the computational cost, the generated network is confined to 72 nodes along the in-plane $X$ direction and 12 nodes along the through-plane $Y$ direction, equivalent to $1800 \mu \mathrm{m} \times 275 \mu \mathrm{m}$ in the $X$ and $Y$ directions, respectively. The pore diameters in the generated network are comparable to commercial GDL pore diameters, while the porosity and permeability are exactly in the desired range, thus the extracted results are expected to be reliable. The network has been depicted in Fig. 2. As can be spotted in the figure, only a slice of GDL which is located under a repeating unit (one GC and two halfribs) of the bipolar plate is considered as the simulation domain. Liquid water is injected from below (GDL/CCL interface), passes through GDL thickness and is discharged into GC.

Considering the stochastic nature of network modeling, resorting to only one generated network for simulation could lead to erroneous and unrealistic results; therefore, a large number of distinct random 


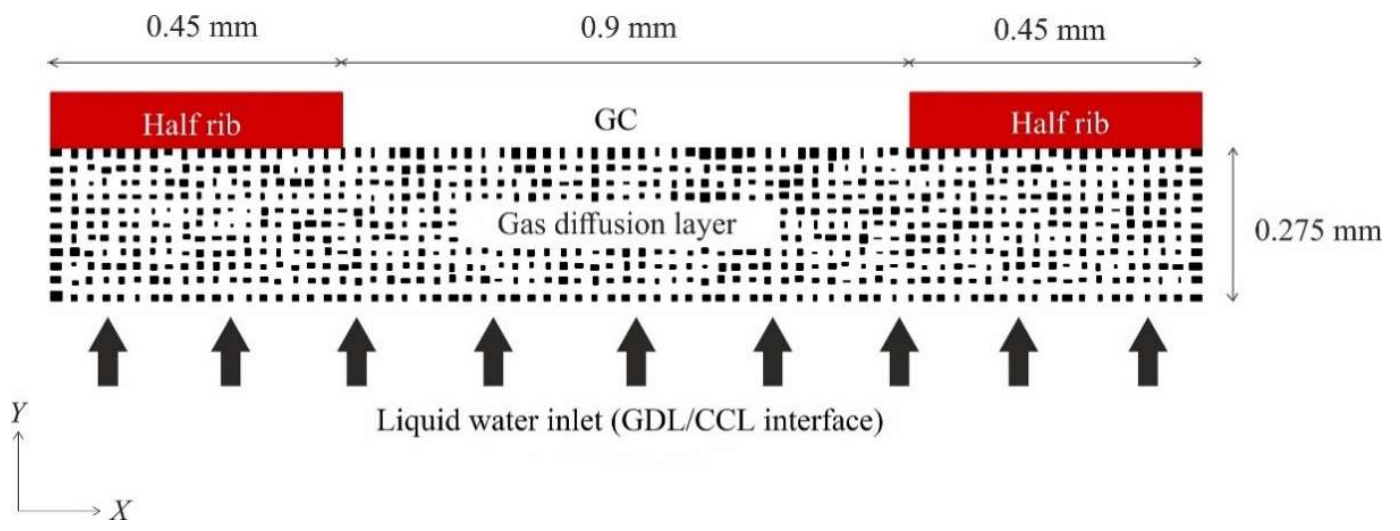

Fig. 2. Schematic of the simulation domain under one repeating unit of the bipolar plate. The nodes which are the centers of each void space are located at the vertices of equal-sized squares which is typical of structured networks. The horizontal or vertical distance between any two neighboring black blocks (solid fibers) represents actually the pore diameter which is twice the defined pore size.

GDL configurations must be generated each possessing the same distribution pattern of Fig. 1, but having different pore size arrangements (realizations); one of the generated pore arrangements is the network portrayed in Fig. 2. All networks will have an identical average pore size, and they will be adjusted to exhibit precisely an identical porosity, too. This is carried out as follows. First, the void volume $\left(V_{\mathrm{P}}\right)$ and the total volume $\left(V_{\text {tot }}\right)$ of the network are calculated. The nominal void volume is determined as $V_{\mathrm{P}, \mathrm{n}}=\varepsilon_{\mathrm{n}} \times V_{\text {tot }}$, in which $\varepsilon_{\mathrm{n}}$ is the nominal or desired porosity. Finally, the modification factor is calculated as $\Omega=V_{\mathrm{P}, \mathrm{n}} / V_{\mathrm{P}}$

. This modification factor is multiplied by each pore volume in the network which renders the network of the desired porosity. The incorporation of a large number of pore arrangements is required to suppress the unreliability involved with the random microstructure of the networks.

\section{GOVERNING EQUATIONS}

The generated water at CCL invades GDL. Similar to almost all the previous works, it is assumed that water invades and travels across GDL in liquid phase which is a logical presumption at high fuel cell relative humidity and high current densities (Caulk and Baker 2010). In the course of invasion, liquid water (non-wetting phase) displaces air (wetting phase) across GDL (immiscible displacement) which is called drainage as opposed to imbibition. It is assumed that the water/air meniscus moves inside the pores according to piston-type motion while ignoring snap-off which is a common practice in drainage simulations (Lenormand et al. 1983; Laurindo and Prat 1996). With respect to the volume of liquid water that runs through GDL at average fuel cell current densities and the viscosity of the fluids involved, the flow regime is capillary fingering for two-phase flow in accordance with the phase diagram of Lenormand et al. (1988). In simpler words, the menisci adopt the routes with the least resistance and select larger ducts while passing through GDL. A capillary threshold pressure is defined for each pore, and obviously the pressure drop across the meniscus must surpass this entry pressure in order for the meniscus to enter the pore, namely:

$$
P_{\mathrm{c}}=P_{\mathrm{w}}-P_{\mathrm{a}}=\frac{2 \gamma \cos \theta_{w}}{R_{\mathrm{P}}}
$$

where $\gamma$ is the surface tension the $\theta_{w}$, is the contact angle of the non-wetting phase which is considered to be $135^{\circ}$, and $R_{\mathrm{P}}$ is the radius of the inscribed circle within the duct defined in Eq. (1); $R_{\mathrm{P}}$ is deemed to be exactly the same as the average radius of curvature necessary for menisci to be allowed into hydrophobic ducts (Lee et al. 2010). After invading a duct, water moves the air along the duct length, however the air maintains film connectivity along the corners of the quadrilateral pore; by that means, the water flows through the bulk of the pore whereas the air moves in the corners (Singh and Mohanty 2003). To justify the presence of corner film flows, the wettability of air must be quite high. The contact angle of air can be calculated as $\theta_{\mathrm{a}}=180^{\circ}-\theta_{\mathrm{w}}=45^{\circ}$. In a square duct, the maximum wetting phase contact angle for the existence of corner films is $45^{\circ}$, thus the wetting films of air do exist in the corners (Chapuis and Prat 2007). No computations of film flows have been contained in the model, neither in terms of pressure nor saturation, however this assumption of quadrilateral pores instead of cylindrical ones and the ensuing corner air flows alter the mixing and merging algorithms when two or more menisci move towards a common node in the network. One of the main outcomes is the fact that trapped air is not formed within the network following the water invasion which is much closer to reality. Dealing with the trapping issue is much more severe in $2 \mathrm{D}$ networks as compared to 3D ones in drainage, because in $3 \mathrm{D}$ networks due to enhanced pore connectivity trapping is less likely to occur (Laurindo and Prat 1996; Vorhauer et al. 2015). 
In the present model, the ducts contain volume with the meniscus moving inside; as a result, capillary pressure inside the duct is not constant and depends on the meniscus position. The capillary pressure model recommended by Médici and Allen (2010) (Eq. (3) in their paper) is compatible with this situation, thereby it will be employed by the present model. As a result, capillary pressure shows a smooth variation inside the duct while the maximum capillary pressure can take place at either the nodes or some position within the duct based on the distribution of the surrounding pore sizes.

Air flow cross-section which is the sum of the area of the four corners of a duct is derived as (Lee et al. 2010):

$A_{\mathrm{a}}=\frac{1}{4} R_{\mathrm{P}}^{2}(4-\pi)$

More precise relations compared to Eq. (3) can be found in literature, however, this equation satisfies the purpose of this paper. Consequently, the water flow cross-section is simply:

$$
A_{\mathrm{w}}=A_{\text {duct }}-A_{\mathrm{a}}
$$

where $A_{\text {duct }}$ is the cross-section of the quadrilateral pore. The viscous flow within the pore is formulated by Washburn equation which is a modified HagenPoiseuille equation to include capillary pressure and two-phase flow effects; the volumetric flow rate inside the quadrilateral pore from node $i$ to an adjacent node $j$ is expressed as (Singh and Mohanty 2003):

$$
Q_{i j}= \begin{cases}\frac{R_{\mathrm{P}}{ }^{2} A_{\mathrm{w}}}{8 \mu_{i j, \mathrm{eff}} L}\left(P_{i}-P_{j}-P_{\mathrm{c}, i j}\right) & , P_{i}-P_{j}>P_{\mathrm{c}, i j} \\ 0 & , P_{i}-P_{j}<P_{\mathrm{c}, i j}\end{cases}
$$

in which $P_{i}$ and $P_{j}$ denote fluid pressures at nodes $i$ and $j$, and $P_{\mathrm{c}, i j}$ is the capillary pressure within the pore. As it can be inferred from Eq. (5), when the pressure drop across the pore exceeds the pore capillary pressure, the meniscus advances forward, otherwise it stops, meaning that reverse flow is not taken into consideration. This is a logical presumption in fuel cell diffusion media, because water is being continuously generated, and in addition, the twisted passageways of actual GDL along with moderate contact angles cause the meniscus not to move back (Tranter et al. 2018). If only one phase exists inside the pore, then $P_{\mathrm{c}, i j}=0$, and $\mu_{\mathrm{eff}, i j}$ will be the viscosity of that phase. If a meniscus exists in the pore, the effective viscosity $\mu_{\text {eff }, i j}$ will be calculated as the saturation weighed average of the viscosity of the two phases, namely:

$$
\mu_{\mathrm{eff}, i j}=\mu_{\mathrm{w}} \frac{X_{i j}}{L}+\mu_{\mathrm{a}}\left(1-\frac{X_{i j}}{L}\right)
$$

where $X_{i j}$ is actually the meniscus position within the quadrilateral duct; $\mu_{\mathrm{w}}$ is the viscosity of water, and $\mu_{\mathrm{a}}$ is the viscosity of air.
Considering water and air as incompressible fluids, mass conservation for node $i$ in the network is expressed as:

$$
\sum_{\mathrm{j}=1}^{4} Q_{i j}=0
$$

Equation (7) results in a linear system of equations for the nodal pressures $\left(\mathrm{P}_{\mathrm{i}}\right)$. The system is solved at every time step by the conjugate gradient method and the flow rates are calculated. All the pores containing a meniscus in which the pressure drop across the pore in the flow direction is lower than the pore capillary pressure are identified and blocked to prevent reverse flow according to Eq. (5), then the pressure field is solved again; this process requires multiple solutions of Eq. (7) at each time step. The time step is determined based on the maximum meniscus velocity that occurs all over the network, namely (Aker et al. 1998):

$$
\Delta t=\min \left[\frac{\Delta x_{\max }}{V_{i j}}\right]
$$

in which, $V_{i j}$ is the meniscus velocity in pore $i j$, and $\Delta x_{\max }$ is the maximum distance the meniscus is allowed to move at each time step which is considered to be $0.4 \mathrm{~L}$ in the model. When approaching the pore ending, the time step is adjusted to allow the meniscus to reach exactly the end of the pore.

Updating the position of the menisci is performed by a second-order Runge-kutta method using the calculated time step as follows:

$$
\begin{aligned}
& X_{i j}= \\
& V\left(X_{0}+\frac{1}{2} V\left(X_{0}, t_{0}\right) \cdot \Delta t, t_{0}+\frac{1}{2} \Delta t\right) \cdot \Delta t+X_{0}
\end{aligned}
$$

where $X_{0}$ is the initial position of the meniscus in the pore, and $t_{0}$ is the initial time. Thus, the physical time of the simulation will proceed as:

$t=t_{0}+\Delta t$

The algorithm proceeds until steady state is confirmed.

An in-house FORTRAN 95 code was written, and no commercial software was engaged in the process. Running the program lasted around 30 minutes to approximately 2 hours for one single network (dependent upon the value of steady state saturation) using a single core of a workstation with a core i5-4460 3.20 $\mathrm{GHz} \mathrm{CPU}$ and $8 \mathrm{~GB}$ of RAM.

\section{RESULTS AND DISCUSSION}

The boundary condition used for the GDL/CCL interface (the network inlet) is a uniform pressure boundary condition; in other words, all the inlet pores at GDL/CCL 


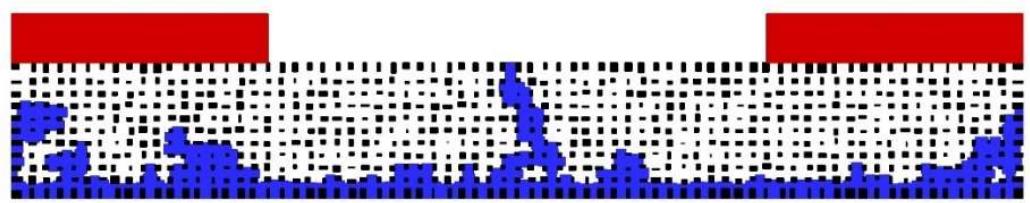

a)

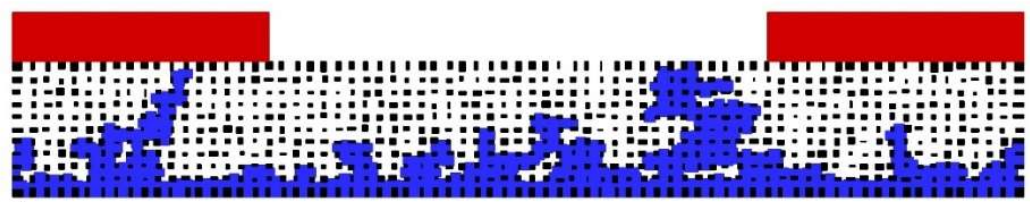

b)

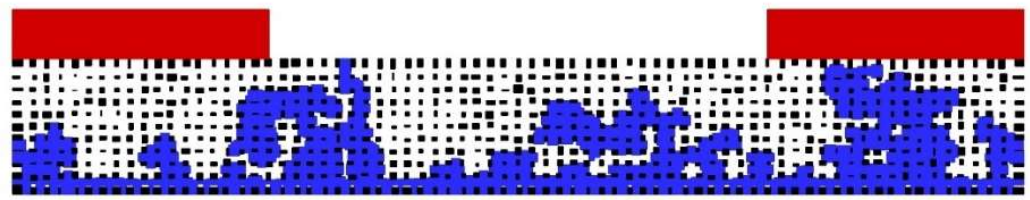

C)

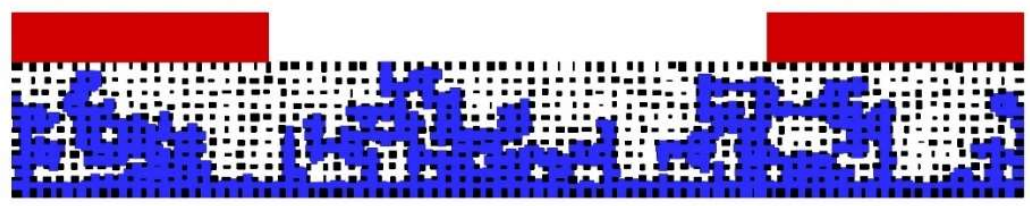

d)

Fig. 3. Steady state liquid water distribution in 4 networks representative of the 30 generated networks. Black colors are the solid fibers of GDL, white colors represent air, and the blue colors represent liquid water. The percolation time of water for each network and the corresponding total saturation for each one are: a) $t=28.0 \mathrm{~s}$ for $S_{\text {tot }}=16.0 \%$, b) $t=49.1 \mathrm{~s}$ for $S_{\text {tot }}=28.01 \%$, c) $t=67.5 \mathrm{~s}$ for $S_{\text {tot }}=38.3 \%$ and d)

$$
t=78.1 \text { s for } S_{\text {tot }}=43.5 \% \text {. }
$$

interface are linked to a reservoir into which water is being injected at a fixed rate. Therefore, regarding the fact that the inlet pore sizes are different, the inlet flow rate will also be different for each pore, resulting in a uniform pressure across the inlet span. To compute the injection flow rate of water into GDL, it is assumed that the fuel cell is working at intermediate current densities; the current density is considered to be fixed at $i=1 \mathrm{~A} / \mathrm{cm}^{2}$. Based on the active area of the GDL under one repeating unit shown in Fig. 2, the total inlet flow rate will be $3.45 \times 10^{-8} \mathrm{~mL} / \mathrm{s}$ which is equivalent to an average of $4.8 \times 10^{-10} \mathrm{~mL} / \mathrm{s}$ for each inlet pore. Assuming the average pore size as determined by the Weibull PDF, the capillary number for this flow would be $1.3 \times 10^{-8}$ which belongs to the capillary fingering regime as postulated by the phase diagram of Lenormand et al. (1988). The boundary condition at the GDL/GC interface in Fig. 2 is set to constant atmospheric pressure, while the boundary condition at GDL/rib interface is set to zero flow rate. Periodic boundary conditions are allocated to the lateral sides of GDL in order to resemble an infinite length in the in-plane direction.
To suppress the uncertainties of network modeling, a total of 30 networks are generated for the base case. The pore sizes in every network are distributed as specified by the frequency distribution of Fig. 1, however, the arrangement of the pore sizes are different. For parametric studies, a total of 20 networks are generated for each case. The network porosities are initially computed, and then tuned to become $\varepsilon=69 \%$. The average permeability of all the networks is calculated as $8.73 \times 10^{-12} \mathrm{~m}^{2}$ along with a standard deviation of $3.11 \times 10^{-12} \mathrm{~m}^{2}$ (VorhauerHuget et al. 2020). The results of simulation will be displayed as the average of the data obtained from all the networks to compensate for the dispersions as a result of pore size arrangements.

\subsection{The Base Case}

The liquid water distribution in GDL at steady state for 4 networks out of the 30 generated networks have been shown in Fig. 3. As can be spotted in the figure, water distribution is different for each network, because pore size arrangements are different. As explained earlier, water is being injected into GDL at a fixed rate; thereby, pushing through the inlet span, the water/air meniscus enters larger pores, and consequently a multitude of the inlet pores do not 
allow the meniscus to enter, as can be seen in the 4 networks. Upon entering the network, water moves chaotically in different directions trying to find easier routes across the network; as a result, although the main injection flow rate is oriented along the through-plane $Y$ direction, the net flow rate along the through-plane direction from CCL towards GC is much smaller. Meanwhile, the majority of intersecting liquid water fronts are merged together causing air clusters to emerge which are actually interconnected through the air films. As soon as water reaches $\mathrm{GC}$, water flow in many of the regions stops, and none of the menisci move, since water has found its route across GDL, and steady state has materialized. During steady state, the dead-end water fingers spotted in Fig. 3 are stationary and do not contribute to water transport across GDL. Within inlet regions of the network, the flow rate is small in the pores containing water, however, as we proceed towards the outlet regions, the flow rates of water will be higher in the pores as a result of water fronts merging together, and finally the whole injection flow rate passes through a single pore at the outlet of the network. The time duration of water percolation will be different for each network; the more tortuous the water percolation routes, the higher the percolation time, and more liquid fingers and higher volumes of water will remain in the network at steady state.

Mean liquid water saturation profiles along the through-plane direction and their evolution towards steady state have been portrayed in Fig. 4. Each curve in the figure is actually the average of the saturation profiles of all the 30 generated networks at the specified time. As can be spotted in the figure, at the time $t=5 \mathrm{~s}$, water has not advanced any further than $Y=125 \mu \mathrm{m}$, however, as time passes, water diffuses into GDL final layers and reaches its maximum during steady state.

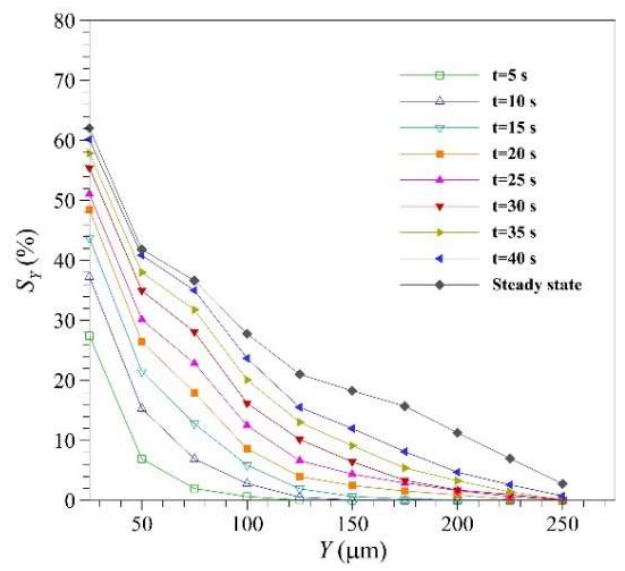

Fig. 4. Temporal evolution of liquid water local saturation profiles along the through-plane direction.

The profiles in Fig. 4 exhibit concave shapes which is typical of saturation profiles in capillary fingering regime either in GDLs or other application areas (Thompson 2002; Singh and Mohanty 2003) which again confirms the compatibility of our results with the phase diagram of Lenormand et al. (1988). At steady state, local saturation of water varies from $S_{Y}=62.1 \%$ at the inlet boundary to $S_{Y}=2.9 \%$ at the outlet boundary with a total saturation of $S_{\text {tot }}=26.3 \%$. The time at which the percolation reaches steady state ranges from $25.7 \mathrm{~s}$ to $78.1 \mathrm{~s}$ for different networks, however $75 \%$ of the networks reach steady state one by one after the time $t=40 \mathrm{~s}$. The curve representing steady state in Fig. 4 is actually the average of saturation profiles of all the networks at steady state that occur at different times. Figure 5 compares our steady state saturation profile with the experimentally determined saturation profiles of Chevalier et al. (2016) for relative humidity in GC equal to $R H=75 \%$ and $R H=100 \%$ and a current density of $i=1.5 \mathrm{~A} / \mathrm{cm}^{2}$. The fuel cell temperature in their experiment was held constant at $60^{\circ} \mathrm{C}$, while the properties used in our model are calculated for cell temperature of $80^{\circ} \mathrm{C}$.

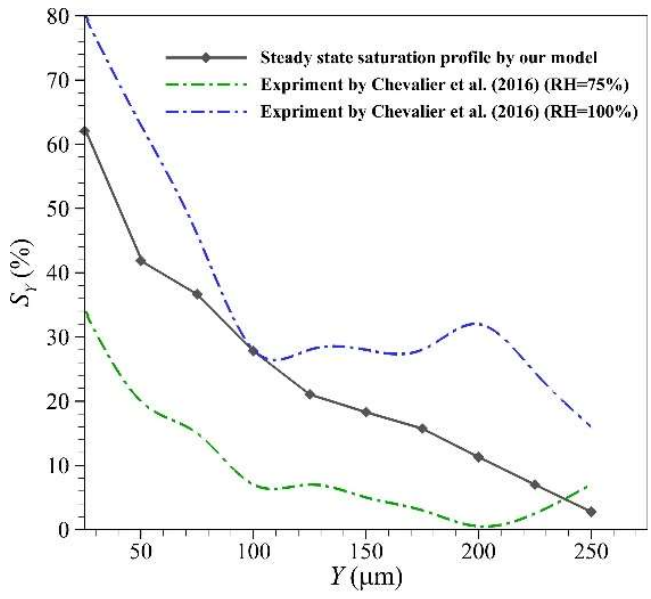

Fig. 5. Comparison of the generated steady state saturation profile with the experimentally determined saturation profiles of Chevalier et al. (2016).

Relative humidity is not an implemented parameter in our model, however, we can say that our saturation profile is somehow equivalent to the case of $R H=100 \%$ (fully humidified blown air), because if the present two-phase flow model were to be coupled to a vapor diffusion model via an isothermal phase change model, the resulting saturation profile would not be altered for $R H=100 \%$ in GC, as shown in Qin (2015); the reason is the lack of vapor concentration gradients. On the other hand, the current density in the work of Chevalier et al. (2016) is higher than the one used in our model. According to X-ray radiography images of Deevanhxay et al. (2011), increasing current density can result in higher saturation levels in GDL. Thus, in the light of the above-mentioned facts and as can be seen in Fig. 5 the general trends are the same and the overall agreement of the results is satisfactory. Lowering relative humidity in GCs or dry gas feeding to fuel cell can lead to drying of liquid fingers in GDL and 
consequently lower saturation. The lower the relative humidity in GCs, the greater the vapor concentration difference between water/air menisci and GC, and the higher the drying rate. Evaporation can cause the liquid fingers not to be capable of breakthrough and reaching GC as shown in Médici and Allen (2013). Temperature gradients are also responsible for phase change phenomena in porous media and GDL (Vorhauer et al. 2018; Surasani et al. 2010), however, they are not incorporated in the present model.

Figure 6 depicts the temporal evolution of capillary pressure profiles along the GDL through-plane direction. The capillary pressure shown on the $Y$ axis is approximately the same as liquid phase pressure, since the pressure in liquid phase is some orders of magnitude higher than the gas phase pressure. As can be seen in the figure, at the time $t=5 \mathrm{~s}$, the pressure is infinitesimal after $Y=125 \mu \mathrm{m}$, because the pores are filled with air in those regions, however, as time passes, water diffuses into GDL final layers and gives rise to pressures. The portrayed pressure at each layer of GDL is actually the average pressure of the nodes in one row of the network, thereby, the pressure at the first row which is totally occupied by liquid water is considerably higher. In the second row, since many of the pores are not invaded by water, the pressure experiences an abrupt drop, however as we proceed towards the outlet boundary, there will be a gradual decrease of water-filled pores and a mild drop in pressure will be experienced.

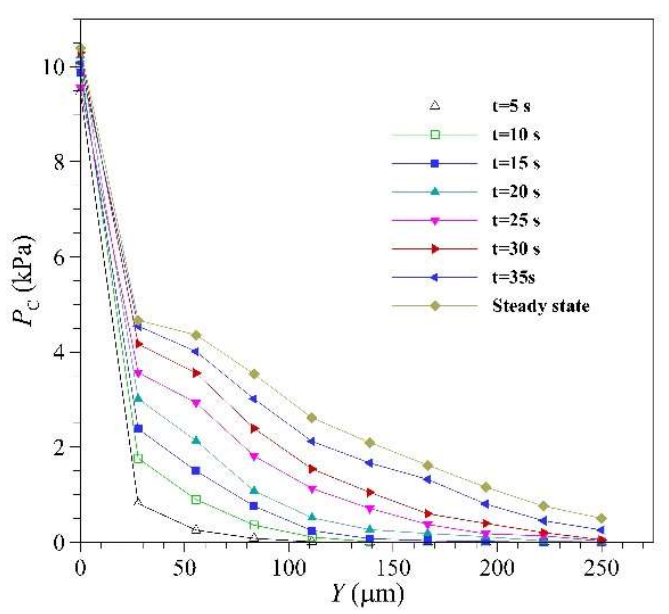

Fig. 6. Time evolution of capillary pressure profiles along the through-plane direction within GDL.

\subsection{Effect of GDL Thickness}

Gas diffusion layer thickness is one of the design parameters in PEFCs. A thick GDL results in high mass transport losses and higher concentration overvoltages, on the contrary, a thin GDL can have a low mechanical support for catalyst layer and may not be able to properly distribute the reactants to reaction sites, thereby, an optimum thickness must be drawn out of PEFC design. In the present work, in order to investigate the effect of GDL thickness, the GDL will be studied at two thicker cases and also two thinner cases compared to the base case. The thicknesses of GDL $(H)$ under study are: $175 \mu \mathrm{m}, 225 \mu \mathrm{m}, 275 \mu \mathrm{m}$ (the base case), 325 $\mu \mathrm{m}$ and $375 \mu \mathrm{m}$, while other network dimensions are the same as before.

Figure 7 depicts one network representative of the 20 generated networks for each thickness $(H)$ displaying the water distributions at steady state. At a first glance, it seems that following a decrease in GDL thickness, the local as well as total saturation decrease in the network. The total amounts of saturation for each case have been calculated which are: $S_{\text {tot }}=19.9 \%$ for $H=175 \mu \mathrm{m}, S_{\text {tot }}=23.9 \%$ for $H=225 \mu \mathrm{m}, \quad S_{\text {tot }}=26.3 \% \quad$ for $H=275 \mu \mathrm{m}$, $S_{\text {tot }}=22.8 \%$ for $H=325 \mu \mathrm{m}$ and $S_{\text {tot }}=22.0 \%$ for $H=375 \mu \mathrm{m}$. As can be understood, for the GDL thicknesses lower than the base case, increasing GDL thickness gives rise to the total saturation, whereas for the GDL thicknesses higher than the base case, increasing GDL thickness results in a mild drop in total saturation. In thin GDLs, the search of water fronts in finding the way out is the lowest, and the routes to outlet are less tortuous leading to lower water accumulation and consequently lower total saturation in the network. In the GDLs thicker than the base case, the abovementioned fact is still true meaning that the search for outlet routes intensifies, however on the other hand, following the enlargement of the network, the network empty volumes expand too, and as a result, the total saturation either is mildly decreased or plateaus. To the best of authors' knowledge, these findings have not been investigated through experimental work before, however, we can compare the results for GDL thicknesses lower than the base case with the simulation of Jeon (2020) which is carried out using lattice Boltzmann method. In the mentioned paper, the GDL thickness of the range $100 \mu \mathrm{m}-300 \mu \mathrm{m}$ has been studied showing that water saturation increases as GDL thickness increases which is in agreement with our findings. Lee et al. (2014) have also examined the effect of GDL thickness via pore network modeling. One of their findings was that in thicker GDLs $(H>300 \mu \mathrm{m})$ increasing GDL thickness has minor effects on saturation level which is in agreement with the results of our model. The present model captures both the ascending and descending saturation level with increasing GDL thickness which is a unique feature. To illustrate this feature, saturation profiles of liquid water along GDL through-plane direction at steady state are compared for the 5 set of networks with different thicknesses in Fig. 8. The abscissa has been made dimensionless to clarify the comparison. As can be observed in the figure, the base case exhibits almost higher local saturation at any layer compared to GDLs with higher or lower thicknesses which leads to a definitely higher total saturation for the base case. 

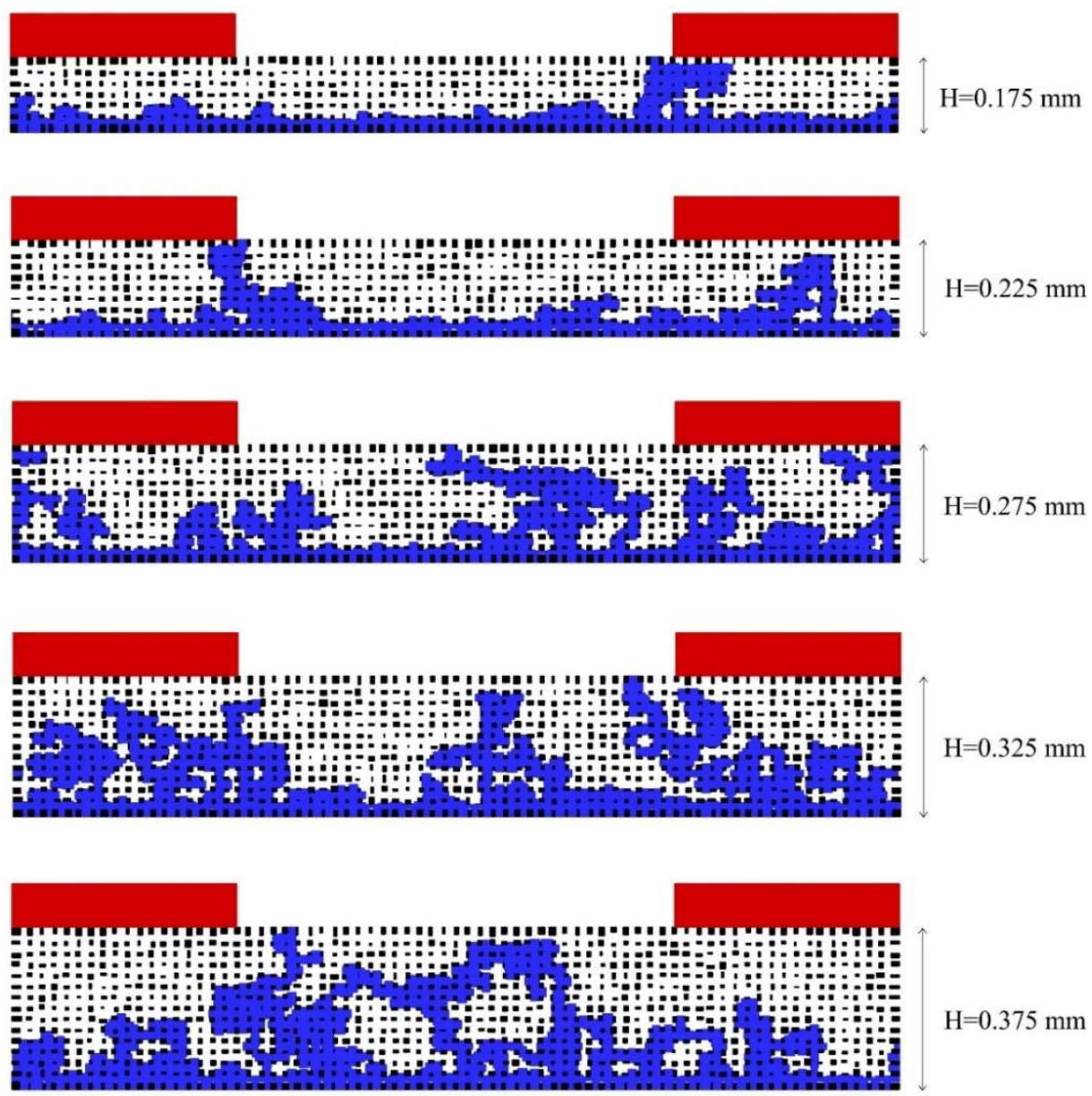

Fig. 7. Steady state liquid water distribution in 5 networks each representing GDL with a different thickness.

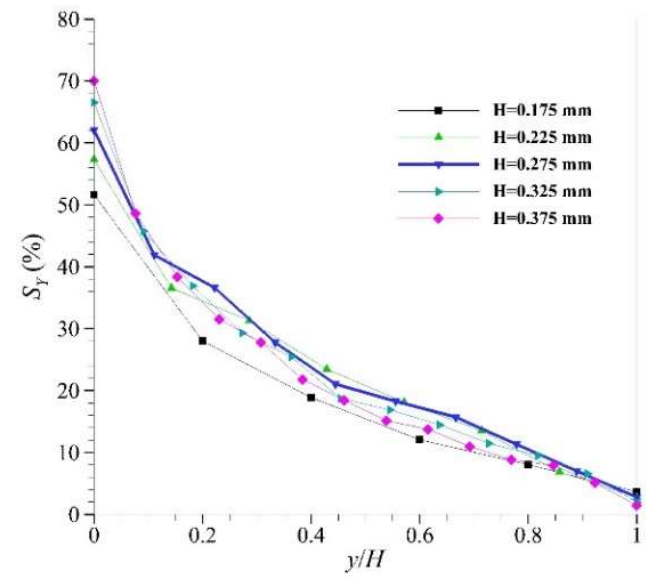

Fig. 8. Steady state liquid water local saturation profiles along the through-plane direction for GDLs with different thicknesses.

Figure 9 portrays capillary pressure profiles along the GDL through-plane direction for the 5 networks of different thicknesses. As can be spotted, at

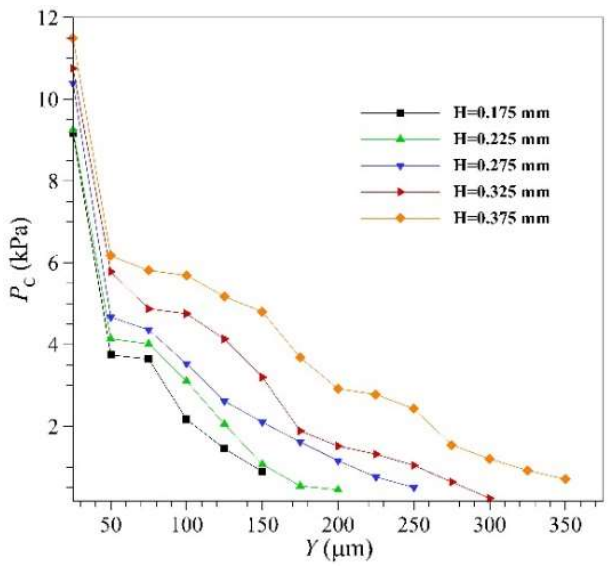

Fig. 9. Steady state capillary pressure profiles along the through-plane direction for GDLs with different thicknesses.

identical distances from the inlet boundary, thicker GDLs have higher fluid pressures, and for thick GDLs, this differences in 

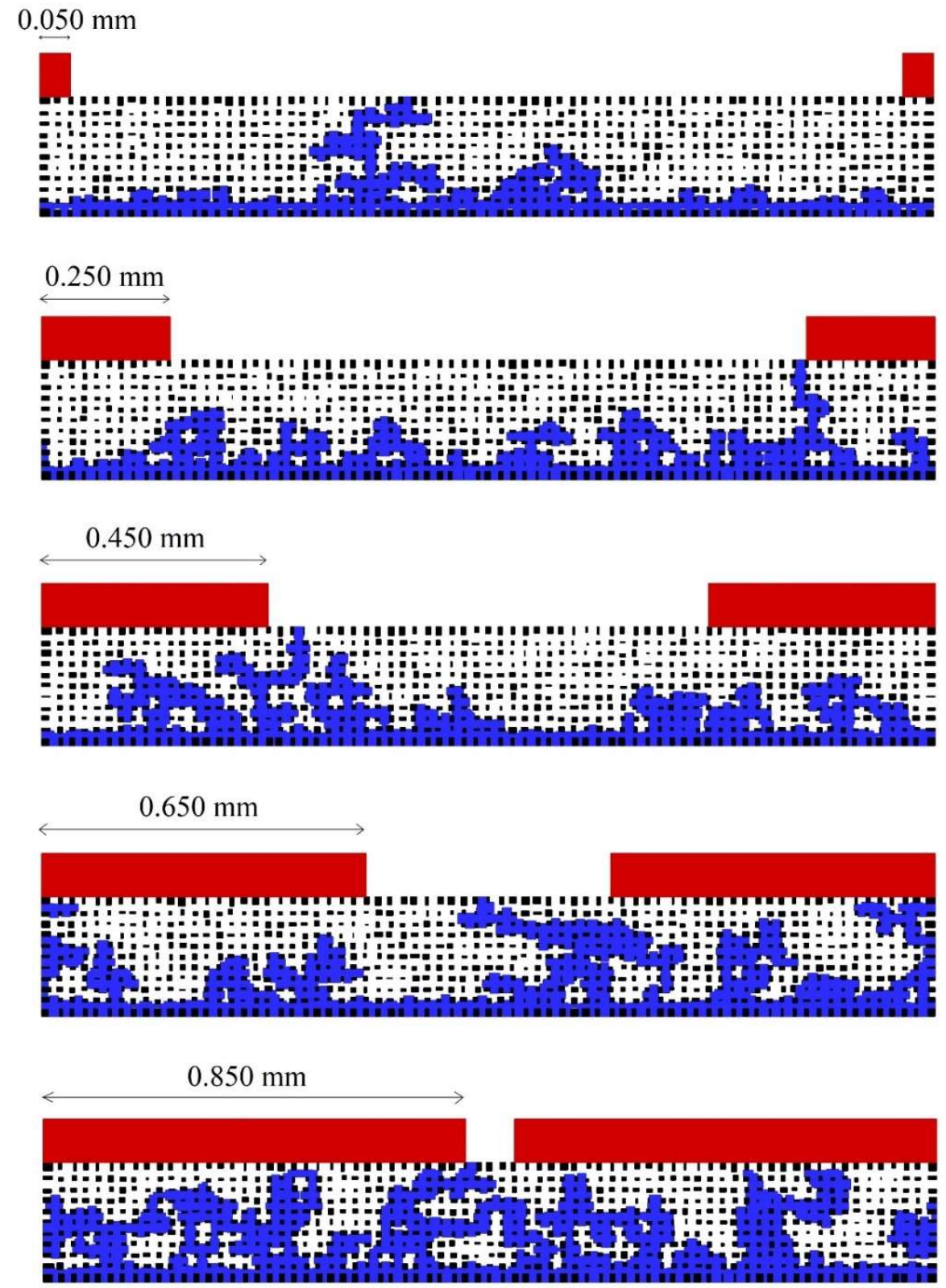

Fig. 10. Steady state liquid water distribution in 5 networks each representing GDLs with different widths of the current collector rib.

local pressures even get larger which can be explained as follows. In thicker GDLs, the water route across GDL lengthens creating more resistance to flow which gives rise to the viscous pressure drop, as a result, the capillary pressure which is roughly equal to liquid pressure increases.

\subsection{Effect of Current Collector Rib Width}

The width of current collector ribs and also their width relative to GC width is one of PEFC design arameters which can have substantial impacts on fuel cell performance. The design of GC flow fields in bipolar plates which is a broad area of research (Wang 2015) cannot be achieved without a thorough understanding of the effect of rib geometry and width on liquid water distribution in GDL. To explore the influences of rib width, 5 different rib widths have been considered for study. The sum of two half-rib widths (abbreviated as " $R i b$ " in the figure legends) have been considered for each case equal to $100 \mu \mathrm{m}$, $500 \mu \mathrm{m}, 900 \mu \mathrm{m}$ (the base case), $1300 \mu \mathrm{m}$ and 1700 $\mu \mathrm{m}$, while other network dimensions have not been altered. Figure 10 shows one generated network for each rib width and the resulting water distribution at steady state. As it is obvious in the figure, following the widening of the half-ribs, water accumulation in the network exacerbates and the total saturation increases. The total saturation for each case has been calculated as: $S_{\text {tot }}=21.0 \%$ for $\quad R i b=100 \mu \mathrm{m}$, $S_{\text {tot }}=21.9 \%$ for $R i b=500 \mu \mathrm{m}, S_{\mathrm{tot}}=26.3 \%$ for $R i b=900 \mu \mathrm{m}, S_{\text {tot }}=30.4 \%$ for $R i b=1300 \mu \mathrm{m}$ and $S_{\text {tot }}=35.3 \%$ for $R i b=1700 \mu \mathrm{m}$. For a better comparison, local water saturation distributions 
along the through-plane direction for different rib widths are depicted in Fig. 11. As it is obvious from the results, the extension of half-ribs gives rise to the local as well as total saturation in GDL. The reason of the phenomenon can be interpreted as follows. As a result of the extension of the half-ribs, the distance between under-rib regions and the network outlet (GC) increases, therefore, water is forced to travel longer in the in-plane direction to reach the outlet, meanwhile making more random movements leaving more stationary water fingers in the network at steady state, as a result, local as well as total saturation are augmented. As can be seen in Fig. 11, the difference between local saturations for the cases $R i b=100$ and $R i b=500 \mu \mathrm{m}$ is small. These cases have the lowest rib widths, and water at many occasions does not choose the under-rib regions for travel, thereby, in most cases water front mixing scenarios and the final saturation distributions are identical for $R i b=100$ and $R i b=500 \mu \mathrm{m}$.

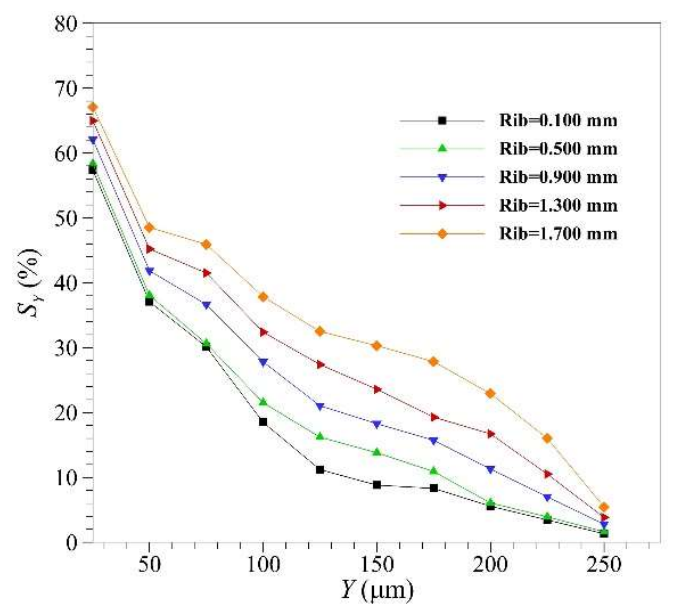

Fig. 11. Steady state liquid water local saturation profiles along the through-plane direction for GDLs with different widths of the current collector rib.

The capillary pressure profiles along the GDL through-plane direction for different rib widths are depicted in Fig. 12. The GDLs with wider ribs exhibit higher fluid pressures in different layers which is due to more accumulation of water. As can be seen, the networks with wider ribs than the base case have considerably higher pressures at the first layer of the network (the inlet boundary) compared to other networks. This is due to the fact that random movements of water fronts under wide ribs produce more water/air menisci each having an interfacial capillary pressure adding up to the water cluster pressure which is interconnected.

\subsection{D Network}

As a concluding section, two 3D networks are generated to achieve a more factual illustration of water configuration in GDL at steady state, because 3D networks possess higher pore connectivity and also higher permeability as compared to $2 \mathrm{D}$ ones, therefore, additional features in terms of water dynamics and saturation may arise.

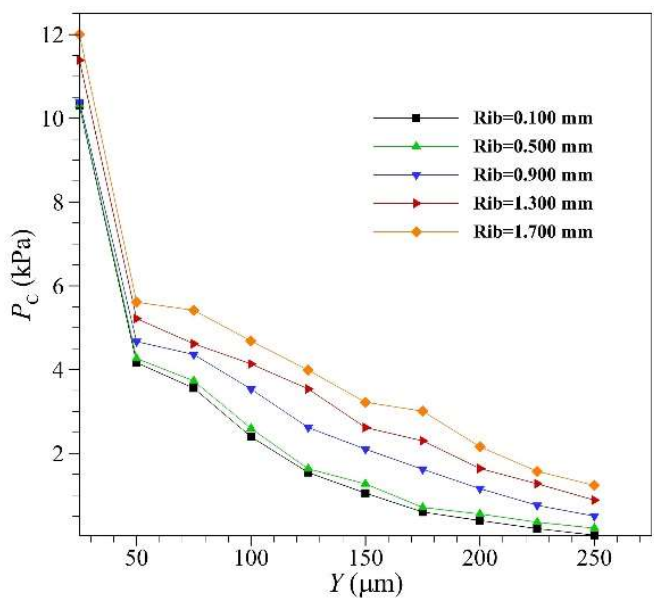

Fig. 12. Steady state capillary pressure profiles along the through-plane direction for GDLs with different widths of the current collector rib.

The network dimensions along the in-plane and through-plane directions are set to be identical to the aforementioned 2D networks. However, due to high computational costs, the number of the pores are made smaller while the pore sizes are made larger resulting in exactly the same porosity as the $2 \mathrm{D}$ networks. The 3D networks are made up of 32 nodes along the in-plane $X$ direction, 8 nodes along the inplane $Y$ direction and 6 nodes along the throughplane $Z$ direction, equivalent to $1800 \mu \mathrm{m} \times 450$ $\mu \mathrm{m} \times 281 \mu \mathrm{m}$ along the $X, Y$ and $Z$ directions, respectively. The pore length is equal to $L=56.25 \mu \mathrm{m}$ throughout the network. The pore size distributions are according to the same Weibull PDF of Eq. (1) with $\xi=5, \eta=12$ and $\beta=2.3$. Based on these values, the pore sizes are dispersed in the range $(5 \mu \mathrm{m}, 35 \mu \mathrm{m})$, and the average pore size of the whole network is $15.5 \mu \mathrm{m}$; the distribution trend is analogous to Fig. 1. The injection flow rate is the same as for $2 \mathrm{D}$ networks for each inlet pore. Running one of the $3 \mathrm{D}$ cases lasts about 10 hours on the mentioned workstation. The $3 \mathrm{D}$ view of liquid water distribution is portrayed in Fig. 13 for the two generated networks. The liquid water fronts which appear fallen apart are a result of the periodic lateral boundary conditions which are now more distinguishable in the $3 \mathrm{D}$ view. The average percolation time is $t=40.4 \mathrm{~s}$ for an average total saturation of $S_{\text {tot }}=20.7 \%$; for the 2D networks, this total saturation corresponds to roughly $t=36.5 \mathrm{~s}$ of percolation time, meaning that water takes longer to reach GC in the 3D networks, since there is an added dimension in the in-plane direction giving rise to the water random movements and the mixing an merging scenarios; this extended percolation time even intensifies at higher levels of saturation. Consequently, the net flow rate along the throughplane direction from CCL towards GC which was mentioned in section 4.1, is smaller compared to the 2D networks. 


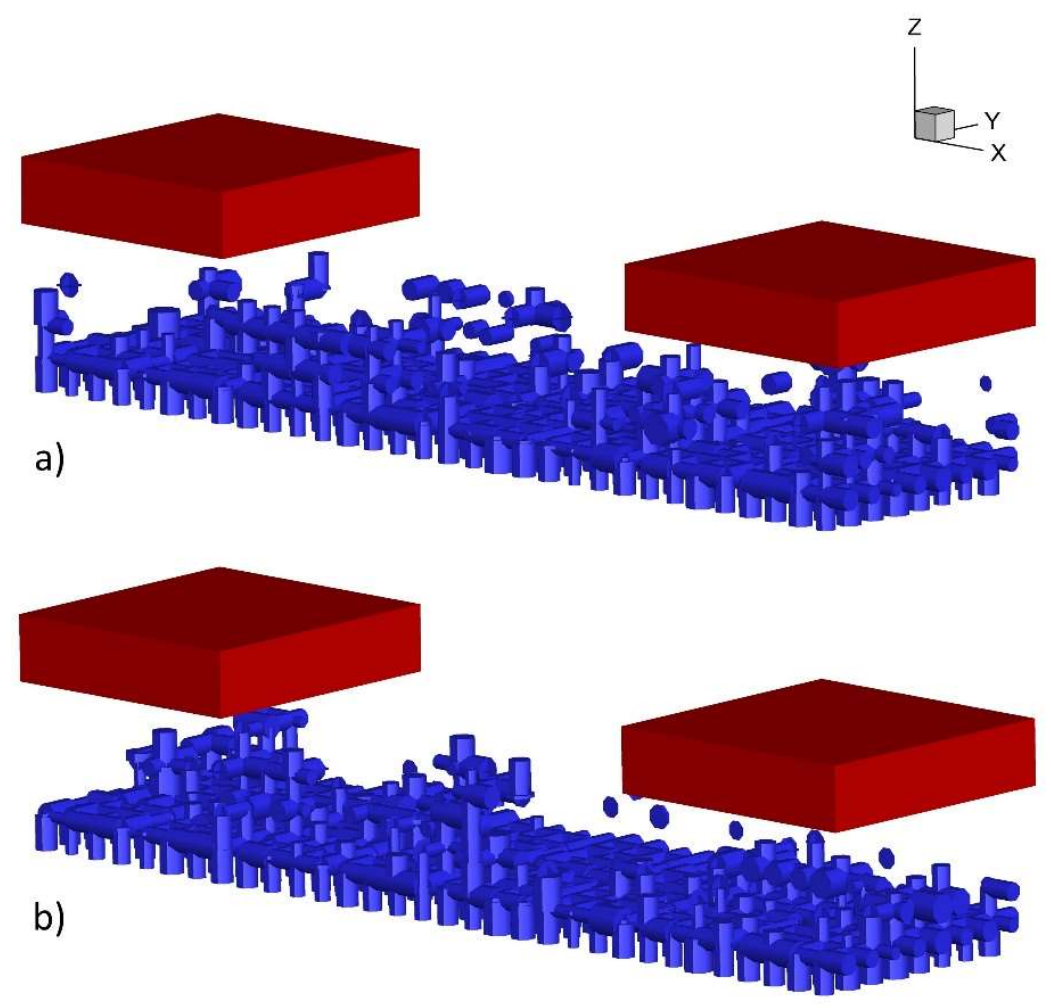

Fig. 13. 3D illustration of liquid water distribution at steady state under a gas channel and two halfribs for the two generated networks. The blue cylinders represent liquid water in the quadrilateral pores. The percolation time and the corresponding total saturation for each network are: a) $t=41.4 \mathrm{~s}$ for $S_{\text {tot }}=21.2 \%$ and b) $t=39.4$ s for $S_{\text {tot }}=20.3 \%$.

As emphasized earlier, the dominant flow regime is capillary fingering, thus if the simulation had been performed using percolation models based on capillary invasion, the results would have not been significantly different, and it would have consumed lower computational resources too. However, a dynamic pore network model generates more acceptable results in terms of physical time and transient behavior, is easier to integrate to different types of boundary conditions and is also facile to couple to other phenomena. Therefore, to extend the current work in the future, a dynamic pore network model is a more appropriate choice.

\section{CONCLUSION}

To tackle the obstacles into PEFCs commercialization, a deep comprehension of the water management and transport problem in the fuel cell electrode is required. The present work made an effort to shed some light on the water movement dynamics in the cathode GDL of the fuel cell. The employed method was a dynamic PNM applied to the porous GDL with an emphasis on geometric parameters such as GDL thickness and current collector rib width. Increasing GDL thickness appeared to increase water saturation at lower thickness ranges, while it seemed to have almost no effect on GDL total saturation at higher thickness ranges. The base case resulted in higher saturation compared to thin and thick GDLs, meaning that either thickening or thinning the GDL alleviates the flooding issue, however, there are other parameters playing role which restrict the choice of thickness. On the other hand, widening the ribs causes more water accumulation in GDL which exacerbate the flooding conditions. In general, current collector ribs should be constructed with some intermediate width which prevents flooding while minimizing the resistance to electron transport. As an overall finding, on account of water content and flooding issue thin GDLs and narrow ribs are favorable.

The present PNM can be upgraded by some means. The heat of reaction which must be removed can be attended to by a network of solids coupled to the present PNM. Incorporating heat transfer can result in phase change within GDL which is feasible through coupling the current two-phase flow model to a vapor diffusion model via a phase change model, albeit thermal and relative humidity boundary conditions are also involved. Coupling the PNM to the phenomena at other fuel cell layers is another interesting topic which can all be envisaged as future extensions of the current work.

\section{REFERENCES}

Aghighi, M., M. A. Hoeh, W. Lehnert, G. Merle and J. Gostick (2016). Simulation of a full fuel cell membrane electrode assembly using pore 
network modeling. Journal of the Electrochemical Society 163(5), F384-F392 (2016).

Aker, E., K. J. Maloy, A. Hansen and G. G. Batrouni (1998). A two-dimensional network simulator for two-phase flow in porous media. Transport in Porous Media 32, 163-186.

Carrere, P. and M. Prat (2019). Liquid water in cathode gas diffusion layers of PEM fuel cells: Identification of various pore filling regimes from pore network simulations. International Journal of Heat and Mass Transfer 129, 10431056.

Caulk, D. A. and D. R. Baker (2010). Heat and water transport in hydrophobic diffusion media of PEM fuel cells. Journal of the Electrochemical Society 157(8), B1237-B1244.

Chapuis, O. and M. Prat (2007). Influence of wettability conditions on slow evaporation in two-dimensional porous media. Physical Review E 75, 046311 .

Chevalier, S., J. Lee, N. Ge, R. Yip, P. Antonacci, Y. Tabuchi, T. Kotaka and A. Bazylak (2016). In operando measurements of liquid water saturation distributions and effective diffusivities of polymer electrolyte membrane fuel cell gas diffusion layers. Electrochimica Acta 210, 792-803.

Deevanhxay, P., T. Sasabe, S. Tsushima and S. Hirai (2011). Investigation of water accumulation and discharge behaviors with variation of current density in PEMFC by high-resolution soft Xray radiography. International Journal of Hydrogen Energy 36(17), 10901-10907.

Fazeli, M., J. Hinebaugh and A. Bazylak (2015). Investigating inlet condition effects on PEMFC GDL liquid water transport through pore network modeling. Journal of the Electrochemical Society 162(7) F661-F668.

García-Salaberri, P. A., I. V. Zenyuk, A. D. Shum, G. Hwang, M. Vera, A. Z. Weber and J. T. Gostick (2018). Analysis of representative elementary volume and through-plane regional characteristics of carbon-fiber papers: diffusivity, permeability and electrical/thermal conductivity. International Journal of Heat and Mass Transfer 127, 687-703.

García-Salaberri, P. A., J. T. Gostick, G. Hwang, A. Z. Weber and M. Vera (2015). Effective diffusivity in partially-saturated carbon-fiber gas diffusion layers: Effect of local saturation and application to macroscopic continuum models. Journal of Power Sources 296, 440453.

Gholipour, H., M. J. Kermani and R. Zamanian (2021a). Coupled pore network model for the cathode gas diffusion layer in PEM fuel cells. Acta Mechanica Sinica 37, 331-348.

Gholipour, H., M. J. Kermani and R. Zamanian (2021b). Network modeling to investigate the effect of coupling the transport phenomena on water distribution in gas diffusion layers. Amirkabir Journal of Mechanical Engineering, in Press.

Jeon, D. H. (2020). Effect of gas diffusion layer thickness on liquid water transport characteristics in polymer electrolyte membrane fuel cells. Journal of Power Sources 475, 228578.

Laurindo, J. B. and M. Prat (1996). Numerical and experimental network study of evaporation in capillary porous media. Phase distributions. Chemical Engineering Science 51(23), 51715185 .

Lee, K.-J., J. H. Kang and J. H. Nam (2014). Liquid water distribution in hydrophobic gas-diffusion layers with interconnect rib geometry: An invasion-percolation pore network analysis. International Journal of Hydrogen Energy 39(12), 6646-6656.

Lee, K.-J., J. H. Nam and C.-J. Kim (2010). Steady saturation distribution in hydrophobic gasdiffusion layers of polymer electrolyte membrane fuel cells: A pore-network study. Journal of Power Sources 195(1), 130-141.

Lee, K.-J., J. H. Nam and C.-J. Kim (2009). Porenetwork analysis of two-phase water transport in gas diffusion layers of polymer electrolyte membrane fuel cells. Electrochimica Acta 54(4), 1166-1176.

Lenormand, R., E. Touboul and C. Zarcone (1988). Numerical models and experiments on immiscible displacements in porous media. Journal of Fluid Mechanics 189, 165-187.

Lenormand, R., C. Zarcone and A. Sarr (1983). Mechanisms of the displacement of one fluid by another in a network of capillary ducts. Journal of Fluid Mechanics 135, 337-353.

Li, H., Y. Tang, Z. Wang, Z. Shi, S. Wu, D. Song, J. Zhang, K. Fatih, J. Zhang, H. Wang, Z. Liu, R. Abouatallah and A. Mazza (2008). A review of water flooding issues in the proton exchange membrane fuel cell. Journal of Power Sources 178(1), 103-117.

Médici, E. F. and J. S. Allen (2013). Evaporation, two phase flow, and thermal transport in porous media with application to low-temperature fuel cells. International Journal of Heat and Mass Transfer 65, 779-788.

Medici, E. F. and J. S. Allen (2010). The effects of morphological and wetting properties of porous transport layers on water movement in PEM fuel cells. Journal of the Electrochemical Society 157(10), 1505-1514.

O'Hayre, R., S.-W. Cha, W. G. Colella and F. B. Prinz (2016). Fuel Cell Fundamentals. 3rd Edition, John Wiley \& Sons.

Qin, C.-Z., B. Guo, M. Celia and R. Wu (2019). Dynamic pore-network modeling of air-water 
flow through thin porous layers. Chemical Engineering Science 202, 194-207.

Qin, C. (2015). Water transport in the gas diffusion layer of a polymer electrolyte fuel cell: Dynamic pore-network modeling. Journal of the Electrochemical Society 162(9) F1036F1046.

Rebai, M. and M. Prat (2009). Scale effect and twophase flow in a thin hydrophobic porous layer. Application to water transport in gas diffusion layers of proton exchange membrane fuel cells. Journal of Power Sources 192(2), 534-543.

Singh, M., and K. K. Mohanty (2003). Dynamic modeling of drainage through threedimensional porous materials. Chemical Engineering Science 58(1), 1-18.

Surasani, V. K., T. Metzger and E. Tsotsas (2010). Drying simulations of various 3D pore structures by a nonisothermal pore network model. Drying Technology 28(5), 615-623.

Tavangarrad, H., B. Mohebbi, S. M. Hassanizadeh, R. Rosati, J. Claussen and B. Blümich (2018). Continuum-scale modeling of liquid redistribution in a stack of thin hydrophilic fibrous layers. Transport in Porous Media 122 (1) 203-219.

Thompson, K. E. (2002). Pore-scale modeling of fluid transport in disordered fibrous materials. AIChE Journal 48, 1369-1389.

Tranter, T. G., J. T. Gostick, A. D. Burns and W. F. Gale (2018). Capillary hysteresis in neutrally wettable fibrous media: A pore network study of a fuel cell electrode. Transport in Porous Media 121(3), 597-620.

Vorhauer, N., E. Tsotsas and M. Prat (2018). Temperature gradient induced double stabilization of the evaporation front within a drying porous medium. Physical Review Fluids 3,114201 .

Vorhauer N., Y. J. Wang, A. Kharaghani, E. Tsotsas and M. Prat (2015). Drying with formation of capillary rings in a model porous medium. Transport in Porous Media 110, 197-223.

Vorhauer-Huget, N., H. Altaf, R. Dürr, E. Tsotsas and T. Vidakovi'c-Koch (2020). Computational Optimization of Porous Structures for Electrochemical Processes. Processes 8(10), 1205 .

Wang, J. (2015). Theory and practice of flow field designs for fuel cell scaling-up: A critical review. Applied Energy 157, 640-663.

Weber, A. Z., R. L. Borup, R. M. Darling, P. K. Das,... , I. V. Zenyuk (2014). A critical review of modeling transport phenomena in polymerelectrolyte fuel cells. Journal of the Electrochemical Society 161(12) F1254-F1299.

Wu, R., Q. Liao, X. Zhu and H. Wang (2012). Impacts of the mixed wettability on liquid Water and reactant gas transport through the gas diffusion layer of proton Exchange membrane fuel cells. International Journal of Heat and Mass Transfer 55, 2581-2589.

Wu, R., X. Zhu, Q. Liao, H. Wang, Y. Ding, J. Li and D. Ye (2010). A pore network study on the role of micro-porous layer in control of liquid water distribution in gas diffusion layer. International Journal of Hydrogen Energy 35, 7588-7593.

Zhan, N., W. Wu and S. Wang (2019). Pore network modeling of liquid water and oxygen transport through the porosity-graded bilayer gas diffusion layer of polymer electrolyte membrane fuel cells. Electrochimica Acta 306, 264-276 